\title{
The thermodynamic characteristics of systems governing by the weighted sum of nonlinear pairwise interactions
}

\author{
Y. A. Pykh \& I. G. Malkina-Pykh \\ Research Center for Interdisciplinary Environmental Cooperation \\ (INENCO RAS), Russian Academy of Sciences, Russia
}

\begin{abstract}
In this report we summarize the results in the application of the Direct Lyapunov method to the generalized replicator systems with the weighted sum of nonlinear pairwise interactions. These complex systems define the properties of a system composed of objects that are coupled via nonlinear pairwise interactions. It is shown that there exist two types of thermodynamic Lyapunov functions: fitness-like and entropy-like. As an example it will be established that practically all known thermodynamic characteristics may be obtained from entropy-like Lyapunov functions for replicator systems.
\end{abstract}

"The positive time direction is associated with the increase of entropy. Let us emphasize the strong and very specific way in which the one-sidedness of time appears in the second law. According to its formulation it leads to the existence of a function having quite specific properties as expressed by the fact that for an isolated system it can only increase in time. Such functions play an important role in modern theory of stability as initiated by the classic work of Lyapunov. For this reason they are called Lyapunov functions (or functionals).

The entropy $S$ is a Lyapunov function for isolated systems. As shown in all textbooks thermodynamic potentials such as the Helmholtz or Gibbs free energy are also Lyapunov functions for other "boundary conditions" (such as imposed values of temperature and volume).

In all these cases the system evolves to an equilibrium state characterized by the existence of a thermodynamic potential. This equilibrium state is an "attractor" for non-equilibrium states." 


\section{Pairwise interactions: an outlook}

The understanding of the nature of the complex interactions between species is of great importance to the study of ecosystems' functioning. As well as the interactions, the species composition also plays an important role in the dynamics and functioning of the system. Ecology deals with interactions and for modeling many ecological processes it is necessary to include explicitly the dynamic interaction between two or more populations. Consequently, binary interactions, such as predation, parasitism, competition, mutualism and others, constitute foundational concepts in population ecology and hence they occupy an important fraction of most ecology research. The simplest model of a community described by a sequence of pairwise interactions is one in which the per capita reproductive is a sum of effects by other species.

Basic models that form the foundation of these interactions, such as the Lotka-Volterra equations and replicator equations, generally assume that interactions are an intrinsic property of the two interacting species and therefore are governed by their respective densities. That is, parameters determining the strength of interactions between species (the interaction coefficients) effectively are assumed to be independent of the community milieu in which these species are embedded. Consequently, more complex communities can be assembled from these pairwise interactions.

Lotka-Volterra and replicator models have played an important role in ecology since their introduction in the early $20^{\text {th }}$ century. These systems serve as a basis for the development of more realistic models that involve ratio dependent functional responses. The classical model allows for the wide range of pairwise interactions between species such as predator/prey, competing species, and symbiotic relationships. These relations lead to a quadratic system of differential equations.

Frequently, interaction in an economic, social, political or computational context is local in the sense that it consists of pairwise interactions between neighbors. In widely used pairwise matching models, if an object interacts at all in a particular period, it interacts with only one (temporary or permanent) partner. Since the early $90 \mathrm{~s}$, a sizeable literature on pairwise interactions between neighbors has emerged. The novel feature is that direct interaction of an object is confined to his neighbors, frequently but not necessarily a small group, while indirect interaction via a chain of neighbors may occur between any pair of objects. As a rule, it has been assumed that the underlying interaction structure does not change over time. Very valuable insights have been gained from studying pairwise interaction under the assumption of a fixed interaction structure.

In systems composed of many elements, rich and complex behavior can emerge from simple interactions. Indeed, for many systems studied in ecology and biology, we can understand almost everything by thinking just about interactions between pairs of elements.

Recent work has shown that probabilistic models based on pairwise interactions - on the simplest case, the Ising model - provide surprisingly 
accurate descriptions of experiments on real biological networks ranging from neurons to genes (Bialek and Ranganathan [1], Roudi et al. [2]).

\section{Nonlinear pairwise interactions}

One of the important assumptions in Lotka-Volterra and replicator systems is that the functional responses are linear functional responses. Usually additive models for predicting combined interactions effects cannot account for nonlinearities in combined functional response introduced by non-trophic interactions (Ayala et al [3]).

Let us consider the system of $n$ types of interacting objects. It may be species, individuals, agents, traits, particles etc. Let $p_{i}(t)$ is the relative frequency of object $i$ at time $t$. The state of the system at time $t$ is simply the vector $p(t)=\left(p_{1}(t), \ldots, p_{n}(t)\right)$ which is clearly constrained to lie in the standard simplex:

$$
\sigma=\left\{p \in \mathbb{R}^{n}: p_{i} \geq 0, i=1, \ldots, n, e^{T} p=1\right\}
$$

We propose that there are exists the set of probability distributions functions: $f_{i}\left(p_{i}\right), i=1, \ldots, n$, which for any $i$ give us probability of the interaction between object $i$ and any other objects in system under consideration. So, the probability of the pairwise interaction between type $i$ and $j$ will be $f_{i}\left(p_{i}\right) f_{j}\left(p_{j}\right)$.

Also, we propose that there are exists the measure $w_{i j}$ of pairwise interaction strength. So we have an interaction (or community) matrix $W=\left(w_{i j}\right)$. Following (Schneidman et al. [4]) in small window of duration $\Delta$ we received, that dynamic of our state variables governed by the next weighted sum of nonlinear pairwise interactions:

$$
p_{i}(t+\Delta) \propto \sum_{j=1}^{n} w_{i j} f_{i}\left(p_{i}(t)\right) f_{j}\left(p_{j}(t)\right)
$$

Based on these assumptions, and taking into account the normalizing condition: $p(t) \in \sigma$ after simple algebra one can receive the next generalized replicator equations Pykh [5]

$$
\begin{aligned}
& \dot{p}_{i}=h(p) f_{i}\left(p_{i}\right)\left(\sum_{j=1}^{n} w_{i j} f_{j}\left(p_{j}\right)-\right. \\
& \left.-\theta^{-1}(p) \sum_{j, k=1}^{n} w_{j k} f_{j}\left(p_{j}\right) f_{k}\left(p_{k}\right)\right) i=1, \ldots, n
\end{aligned}
$$

Here, $f_{i}$ are nonlinear response functions (probability distributions) satisfying the conditions $f_{i}(0)=0, \partial f_{i} / \partial p_{i}>0 p_{i}>0$, and $\partial f_{i} / \partial p_{i} \geqslant 0$ for $p_{i}=0 ; W=\left(w_{i j}\right)$ is the matrix of interactions; the function $h: \sigma \rightarrow(0, \infty]$ is 
determined by the particular problem under consideration; $\theta(p)=\langle\mathbf{e}, f(p)\rangle$, where $\langle\cdot, \cdot\rangle$ is the inner product; and $f(p)=\left(f_{1}\left(p_{1}\right), \ldots, f_{n}\left(p_{n}\right)\right)$. Obviously, since $\langle\dot{p}(t), \mathbf{e}\rangle \equiv 0$ and $f_{i}(0)=0$, the simplex $\sigma$ and each of its faces are invariant sets of system (1). Note, that condition $f_{i}(1)=1$ is not essential in our consideration.

System (1) has a very wide range of applications, from mathematical genetics and ecology (Fisher [6]) to neural networks and optimization problems (Bomze [7], Morgan and Steiglitz [8], Hofbauer and Sigmund [9]). Recently, it was shown by Helbing [10] that system (1) can be obtained from Boltzmann-like equations. Thus, there are grounds for believing that system (1) determines the evolution of probability distributions for a fairly wide variety of processes.

\section{The main mathematical results}

To state the main theorem, we need some preliminary results. First, it is convenient to pass to the matrix form of representation. In this form, system (1) becomes

$$
\dot{p}=h(p) \mathcal{D}(f)\left(W f-\mathbf{e} \theta^{-1}(p)\langle f, W f\rangle\right)
$$

where $\mathcal{D}(f)=\operatorname{diag}\left(f_{1}, \ldots, f_{n}\right)$.

If the matrix $\mathrm{W}$ is nondegenerate, then system (2) has at most one isolated equilibrium point in $\operatorname{Int} \sigma$, which we call nontrivial.

Statement [5]. System (2) has a unique nontrivial equilibrium point $\hat{p} \in \operatorname{Int} \sigma$ if and only if the vector $W^{-1} \mathbf{e}$ is either strictly positive or strictly negative.

Theorem 1 [5]. If the matrix $W$ is symmetric, then the function

$$
E(p)=\langle f(p), W f(p)\rangle \theta^{-2}(p)
$$

is a Lyapunov energy function for system (2).

Corollary [5]. If system (2) has a nontrivial equilibrium point $\hat{p} \in \operatorname{Int} \sigma$, then it is totally stable in Int $\sigma$ if and only if the matrix $W$ has $(n-1)$ negative characteristic numbers.

Theorem 2 [5]. If $W=W^{T}$ and system (2) has a nontrivial equilibrium point $\hat{p} \in \operatorname{Int} \sigma$ which is totally stable in Int $\sigma$, then the entropy-like function:

$$
H(p)=\sum_{i=1}^{n} \int_{\hat{p}_{i}}^{p_{i}} \frac{\hat{f}_{i} d x}{f_{i}(x)}
$$

is a Lyapunov energy function for system (2), and

$$
\dot{H}=h(p) \hat{\theta} \theta(E(\hat{p})-E(p)) \geq 0 .
$$

Now, we can state the main result without restriction $W=W^{T}$ : 
Theorem 3 [11]. If system (2) has a nontrivial equilibrium point $\hat{p} \in$ Into and the matrix $\left(W^{T}+W\right)$ has $(n-1)$ negative characteristic value, then the function

$$
H(p)=\sum_{i=1}^{n} \int_{\hat{p}_{i}}^{p_{i}} \frac{\hat{f}_{i} d x}{f_{i}(x)}
$$

is a Lyapunov energy function for system (2).

Corollary [11]. If the conditions of Theorem 3 are fulfilled and $W$ is such that $W^{T} W^{-1}$ is a stochastic matrix, i.e.,

$$
W^{T} W^{-1} \mathbf{e}=\mathbf{e},
$$

then the entropy production is defined by the formula

$$
\dot{H}(p)=h(p) \theta \hat{\theta}(E(\hat{p})-E(p)) \geqslant 0
$$

Based on this theorem we can receive a set of response function for existing entropy measures and construct new entropy measures for any response functions. Short summary of this approach listed below in table 1 and 2 .

Table 1: $\quad$ Different entropy measures.

\begin{tabular}{|c|c|c|}
\hline Response function & Entropy & Name \\
\hline $\begin{array}{c}\text { Logarithmic } \\
f_{i}\left(p_{i}\right)=\left(1-\ln p_{i}\right)^{-1}\end{array}$ & $H(p)=\sum_{i=1}^{n} p_{i} \ln p_{i}$ & $\begin{array}{c}\text { Bolzmann } \\
\text { Entropy }\end{array}$ \\
\hline Power-law & $H(p)=\frac{\left(\sum p_{i}^{q}-1\right)}{1-q}$ & $\begin{array}{c}\text { Tsallis } \\
\text { Entropy }\end{array}$ \\
$f_{i}\left(p_{i}\right)=p_{i}^{1-q} ;$ & & $\begin{array}{c}\text { Logistic } \\
\text { entropy } \\
\text { (new) }\end{array}$ \\
\hline$f_{i}\left(p_{i}\right)=\frac{1}{b+c e^{-\alpha p_{i}}}$ & $H(p)=\sum_{i=1}^{n} \ln \left(1-e^{-\alpha p_{i}}\right)$ & ? \\
$b>0, c>0, \alpha>0$ & & \\
\hline
\end{tabular}

\section{Thermodynamic characteristics}

We have received expression (3) for replicator's systems energy, expression (4) for systems entropy and expression (5) for entropy production. On the analogy of thermodynamics laws, we can receive expression for systems temperature. Indeed if we redraft (5) as follows:

$$
\frac{d H}{d E}=h(p) \hat{\theta} \theta(p),
$$

then according to Clausius definition the systems temperature $T$ is equal:

$$
T=(h(\hat{p}) \hat{\theta} \theta(p))^{-1}
$$


Table 2: $\quad$ Different distance measures.

\begin{tabular}{|c|c|l|}
\hline Response function & «Distance» & Name \\
\hline Linear & $H=\sum_{i=1}^{n} \hat{p}_{i} \ln \frac{p_{i}}{\hat{p}_{i}}$ & Relative entropy \\
\hline$f_{i}\left(p_{i}\right)=p_{i}$ & $H=\sum_{i=1}^{n} d_{i} \hat{p}_{i} \ln \frac{p_{i}}{\hat{p}_{i}}$ & $\begin{array}{l}\text { Weighted } \\
\text { relative entropy }\end{array}$ \\
\hline$f_{i}\left(p_{i}\right)=p_{i}$ & $-\langle d, \hat{p}\rangle \ln \langle d, p\rangle$ & $\begin{array}{l}\text { Weighted } \\
\text { logistic entropy } \\
\text { (new) }\end{array}$ \\
\hline Logistic & $H=\sum_{i=1}^{n} \hat{f_{i}} \ln \left(\frac{1-e^{-\alpha p_{i}}}{1-e^{-\alpha \hat{p}_{i}}}\right)$ & \\
\hline$f_{i}\left(p_{i}\right)=\frac{1}{b+c e^{-\alpha p_{i}}}$ & & \\
\hline$b 0, c>0, \alpha>0$ & &
\end{tabular}

Note that in this case the temperature depends from systems steady-state. Now let us consider the exergy of the system. Exergy is a measurement of how far a certain system deviates from a state of equilibrium with its environment. Exergy for a system in an environment usually is written as:

$$
E x=T(\hat{H}-H)
$$

So we have a lot of different expression for exergy dependence from entropy i.e. from response function. If we put:

$$
f_{i}\left(p_{i}\right)=\frac{1}{1-\ln \frac{p_{i}}{\alpha_{i}}}
$$

where vector $\alpha=\left(\alpha_{1}, \ldots, \alpha_{n}\right) \in \sigma$ and interaction matrix is stochastic i.e. $W \mathbf{e}=\mathbf{e}$, then $\alpha_{i}=\hat{p}_{i}$. In this case we receive the next expression for exergy:

$$
E x=h(p) \hat{\theta} \theta(p)\left(\sum_{i=1}^{n} p_{i} \ln \frac{p_{i}}{\hat{p}_{i}}+\left(p_{i}-\hat{p}_{i}\right)\right)
$$

It is easy to see that this expression almost coincide with formula proposed by Mejer and Jorgensen in 1979. Note, that in like manner we can receive all thermodynamic potentials such as Helmholtz or Gibbs free energy, which are also Lyapunov functions. We want to also point out that very similar results may be received for generalized Lotka-Volterra systems with nonlinear functional response (Pykh [12]).

\section{Conclusion}

It is seen from the examples given above that many (and practically all) known entropy characteristics may be obtain from entropy-like Lyapunov function. We also emphasize that there exists a relation between the derivative of the function $H(p)$, which can be interpreted as generalized entropy, and the function $E(p)$, which is often considered as an analog of the energy or fitness. This relationship 
for entropy production was established by Pykh [9] and [8] for different interactions matrix and has the next form:

$$
\dot{H}=h(p) \hat{\theta} \theta(E(\hat{p})-E(p)) \geqslant 0
$$

We mention also that all results stated above were obtained by formally analyzing systems of generalized replicator equations, which arise in very diverse fields of natural sciences and, therefore, can serve as a basis for finding analogies between these domains of natural sciences. Also note that it was Ilya Prigogine who the first pointed out [13] the importance of the relationship between Lyapunov functions and entropy.

\section{References}

[1] Bialek, W. \& Ranganathan, R. Rediscovering the power of pairwise interactions. arXiv: 0712.4397v1 [q-bio.QM], 28 December, 2007.

[2] Roudi, Y., Nirenberg, S., Latham, P.E. Pairwise maximum entropy models for studying large biological systems: when they can and when they cant't work. arXiv: 0811.0903v1 [q-bio.QM], 6 November, 2008.

[3] Ayala, F. J., Gilpin, M. E., Ehrenfeld, J.G. Competition between species: theoretical models and experimental tests. Theor. Pop. Biol., V.4, N.3, pp.331-356, 1973.

[4] Schneidman, E., Berry II, M.J., Segev, R. \& Bialek, W. Weak pairwise correlations imply strongly correlated network states in a neural population. Nature. V.440, pp. 1007-1012, 20 April, 2006.

[5] Pykh, Yu. A. Energy Lyapunov function for generalized replicator equations. Proc. of International Conference "Physics and Control," St. Petersburg, Russia (IFEE Publ., 2003), V. 1, pp. 271-276, 2003.

[6] Fisher, R. A. The genetical theory of nature selection. Clarendon Press: Oxford, 1930.

[7] Bomze, I. M. Evolution towards the maximum clique, Journal of Global Optimization, N 10, pp. 143 - 164, 1997.

[8] Morgan, J., Steiglitz, K. Pairwise competition and the replicator equation. Bulletin of Mathematical Biology, 65, pp. 1163-1172, 2003.

[9] Hofbauer, J. \& Sigmund, K. Bull. Am. Math. Soc. V.40, N 4, pp. 479-519, 2003.

[10] Helbing, D. A stochastic behavioral model and a 'microscopic' foundation of evolutionary game theory. Theory Decision, V. 40, N 2, pp. 149-179, 1996.

[11] Pykh, Yu. A. Construction of the entropy measures on the basis of replicator equation with nonsymmetrical interaction matrix. In Doclady Mathematics. V 72, N 2, pp. 780-783, 2005.

[12] Pykh, Yu. A. Lyapunov functions for Lotka-Volterra systems: an overview and problems. Proc. of 5th IFAC Symposium "Nonlinear Control Systems", pp.1655-1660, 2001.

[13] Prigogine, I. Time, structure and fluctuations. Nobel lecture, 8 December, 1977. 